\title{
Disparity Estimation for Stereo Sequences Based on Adaptive Size Hierarchical Block Matching
}

\author{
Marco Accame and Francesco De Natale \\ University of Genova, Dept. of Biophysical and Electronic Engineering \\ Via Opera Pia 11a, Genova 16135 Italy
}

\begin{abstract}
The paper focuses on a new method for block based disparity estimation (BBDE) that is specially suited for real time coding of stereo sequences. The estimation is performed by exploiting a preliminary disparity field, obtained from the coarsest level of a multiresolution pyramid, and its successive refinements in the finer ones. A strong correction strategy for wrong estimates has been implemented by using an appropriate propagation of disparity vectors from one level to the next one and an adaptive search range. Finally, a variable-resolution disparity field is achieved stopping the propagation at an intermediate level of resolution, if a vector allows a good reconstruction quality of its block.
\end{abstract}

\section{Introduction}

Stereo sequences greatly enhance the effect of presence in visual communication, providing realness by the perception of the third dimension. So their integration in a variety of video applications (such as video-conferencing, video-telephony, digital TV and so on), seems very interesting. A great problem to be solved, is the definition of suitable coding strategies that reduce the large bandwidth required by stereo transmission. Usual video coding techniques, exploiting spatial and temporal redundancy of the scene, may be used to code each sequence independently. However stereovision properties permit considering even cross redundancy (i.e., the similarity between right and left), since two cross-frames result to be locally shifted of an amount called disparity, which is related to the depth of the scene.

This information can be extracted, and then exploited for an efficient crossframe predictive coding (disparity estimation and compensation). In block based approaches (BBDE, BBDC), to find out the disparity vector which will be used for the compensation, a block of pixels from the current right frame is compared with blocks that belong to the related left frame and that fall within a search window along the epipolar line $[1,2,3]$. Usually, a minimum mean square error (MSE) match criterion is used, performed with a full search within the window. In spite of its algorithmic simplicity, the method requires a high computational load that can be hardly managed on line. BBDE presents another major drawback: when the block size is great if compared to objects in the scene, in general it is impossible to achieve a good estimation, for one block may contain several objects at different depth (i.e., different disparity values). This results in false matchings that produce annoying block artifacts 
and evident reconstruction errors. And by reducing block sizes, the computational load is increased and compression efficiency greatly reduced. In the authors' opinion, these intrinsic limitations of fixed size BBDE can be overcome only by using adaptive criteria able to better exploit local characteristics as in [4]; moreover, they have to be used jointly with a multiresolution representation, which allows speeding up the disparity estimation process (see $[5,6]$ for applications to motion estimation).

In a multiresolution context, efficient strategies for propagating and updating disparity vectors permit to recovery from wrong estimates, and to increase the estimation quality, without the addition of redundant disparity field information. In the following, a BBDE technique using a multiresolution representation is described; after, a novel and efficient propagation strategy is presented, which is aimed at defining: (i) if a finer estimation of the disparity inside a block has to be performed; (ii) the value of the relative vector from which the update has to start; (iii) the adaptive searching-window size.

Results achieved by applying our algorithm to a standard test stereo sequence (aqua) are reported at the end, together with a comparative example of BBDEs.

\section{Multiresolution Block-based Disparity Estimation}

A multiresolution BBDE is helpful as, in general, in a scene there are objects covering different scales. Consequently, a matching criterion processing same scale objects together, succeeds in eliminating false matches and achieves a better computational efficiency. There is, indeed, just a small correction of the already processed objects at coarser scales.

For the multiresolution approach, we use a $L$-levels Gaussian pyramid [7], which is a set including the original image $((0)-t h$ level) and its $L-l$ approximations at a dyadic sequence of resolutions. At the (L-I)-th level (lowest resolution), a fixed size BBDE is carried out using a full search in a reduced window along the epipolar line. Each disparity vector is then mapped, on the $(L-2)$-th level, into four new vectors, each one pointing to the starting position of a more accurate estimation. The new vectors describe the disparity of the four $B$-sized blocks, originated by the dyadic twodimensional decimation at this level of the $B$-sized block of $(L-1)$-th level. A coarseto-fine propagation and estimation are then iterated until the $(0)$-th level is reached. Due to the corrective nature at finer levels, a much smaller searching window is needed for matching, so allowing for a considerable reduction in computational load even at these levels. If the previous vector is consistent with the exact disparity, the little correction is satisfactory, otherwise the small window cannot recover from false matching. A specific strategy is then needed, both for the choice of initial vectors and for setting adaptively the width of the updating window. Moreover, if a block covers completely an area with constant disparity and the compensation is satisfactory, it is not necessary to generate other blocks from it.

\section{Propagation Strategy}

The propagation strategy classifies a generic block $b_{B, L-1}(i, j)$ (size $B$ and $(L-1)$-th level) on the basis on the reconstruction error and then: 
- decides if the block needs a more accurate compensation (that is, it requires more than one vector in its inside);

- finds out the four initial vectors for the further disparity estimation phase, together with the relative search window.

In the following, a detailed explanation of the modus operandi is given.

\subsection{Adaptive Size Block Generation}

Each block $b_{B, L-1}(i, j)$ is formed by four sub-blocks of size $B / 2$ at the same $(L-1)$-th level: $b_{B / 2, L-1}(2 i, 2 j), b_{B / 2, L-1}(2 i+1,2 j), b_{B / 2, L-1}(2 i, 2 j+1)$, and $b_{B / 2, L-1}(2 i+1,2 j+1)$. The compensation is evaluated for each one of the sub-blocks; they are compensated with the same disparity vector $d_{B, L-1}(i, j)$ of the block $b_{B, L-1}(i, j)$; if at least one of them has a bad reconstruction, the block $b_{B, L-1}(i, j)$ is split and generates four new son-blocks at the ( $L-2)$-th level, where a new estimation is to be carried out for each block starting from an adequate initial vector. That is, by splitting the father-block $b_{B, L-1}(i, j)$, the four son-blocks are: $b_{B, L-2}(2 i, 2 j), b_{B, L-2}(2 i+1, j), b_{B, L-2}(2 i, 2 j+1)$, $b_{B, L-2}(2 i+1,2 j+1)$ with the associated initial vectors $d_{B, L-2, \text { init }}(2 i, 2 j)$, $d_{B, L-2, \text {,nit }}(2 i+1,2 j), d_{B, L-2, \text {,nit }}(2 i, 2 j+1), d_{B, L-2, \text { init }}(2 i+1,2 j+1)$. Their values are set using the strategy reported in sub-paragraph 3.2.

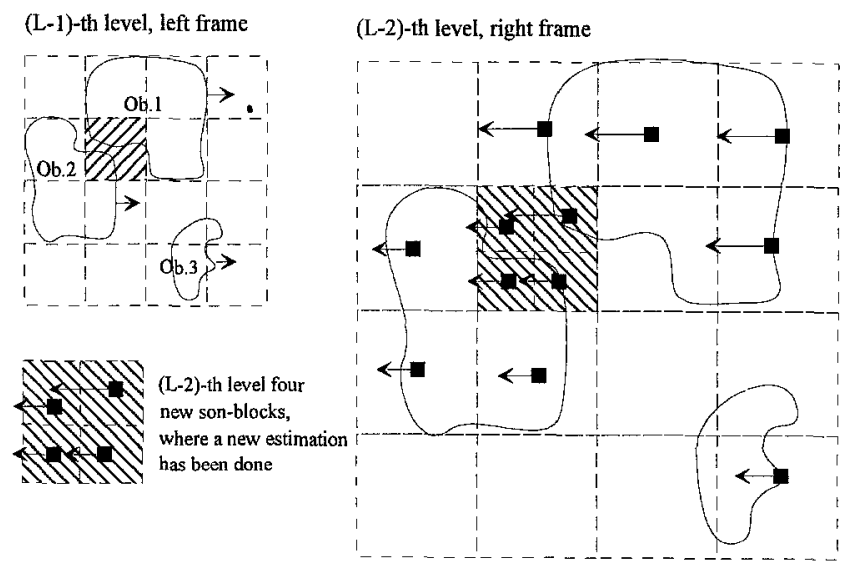

Fig.1. An example of the adaptive size BBDE.

The split and update process is iterated across levels $l \in\{L-1, \cdots, 1\}$, until the reconstruction is satisfactory for every sub-block. If that is reached for e.g., the (l)-th level, then it is checked the compensation of the block $b_{2^{\prime} B, 0}(i, j)((0)$-th level, block size $2^{l} B$ ), done with the best vector $d_{2^{l} B, 0}(i, j)$ within the search window pointed by $d_{2^{I} B, 0, i n i t}(i, j)$, which is the vector $d_{B, l}^{2^{l}, 0}(i, j)$ expanded by a factor $2^{l}$. The leveldependent width of the window is $2^{l}+1$ and gives subpixel accuracy for the $(l)$-th level (see Fig.2). If the compensation is satisfactory the block $b_{B, l}(i, j)$ at the $(l)$-th level is not split anymore in the propagation, and just one vector is used for its disparity description. The mechanism permits to create blocks of different dimensions, which adaptively cover the right frame, and the vector concentration is higher just where a denser disparity field is needed. This kind of block generation is equivalent to a quadtree growing process, whose root is the block $b_{B, L-1}(i, j)$. That 
helps to code very compactly the side information needed to place vectors on the frame. Indeed, it is just necessary to code with one bit each split decision. With this information, it is possible to reconstruct the quadtree and place the blocks on the frame with the relative vectors

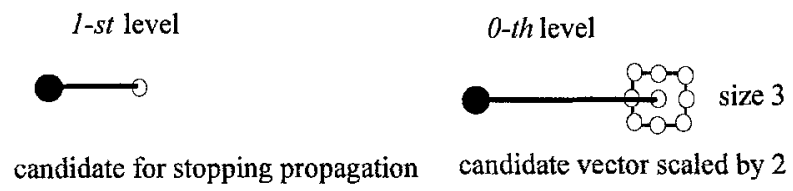

Fig.2. Example of subpixel search window for testing a propagation stop hypothesis.

\subsection{Vector Propagation Strategy}

The propagation of a vector from the (l)-th is different whether the related compensation is satisfactory or not. In the first case the vector $d_{2 l_{B, 0}}(i, j)$, associated to the block $b_{2^{l} B, 0}(i, j)$, is taken directly on the (0)-th level, as seen before. In the other case, four new vectors are propagated to the (l-1)-th level in this way (Fig.4).

- At first each initial vector takes the best scaled vector among the set of its neighbors at the (l)-th level (see [8], and [7] for an interesting application).

- Then, during the updating phase, the vector is again compared to its neighbor ones, but those on the same (l-1)-th level, which have been already updated.

Right frame, (l)-th level Right frame, $(l-l)$-th level

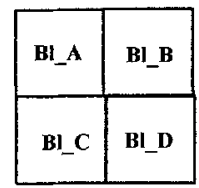

Right frame, (l-1)-th level, during updating

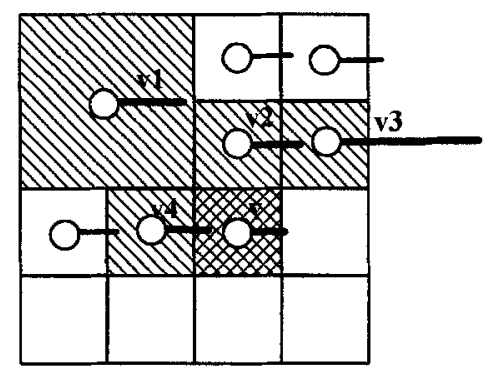

Fig.3. At first the initial vector $v$ for BI_D1 in the $(l-l)$-th level is chosen as the one related to the block set $\left\{B l_{-} A, B l_{-} B, B l_{-} C, B l \_D\right\}$ giving the best compensation. In the next step, the already refined neighbor vectors $\{v 1, v 2, v 3, v 4\}$ and $v$ are checked to get the best one.

\subsection{Adaptive Size Setting for the Search Window}

The initialization of a vector onto the (l-1)-th level, needs to be followed by the search for the one which minimize the MSE of the compensation at that level. If the initial MSE is not too large, but above a fixed threshold (LOW_THR, see Fig.4), it is not necessary to set a large window width; on the contrary, a high MSE means that the initial estimate is not satisfactory, and a new search with large scale is needed. In this work, the width of the search window is set as a piecewise linear function of the initial MSE. The width depends even on the resolution level, so a normalization factor of $2^{-l}$ is applied when the search is done on the $(l)$-th level. Moreover, the 
window is not square and depends on the slope of the epipolar line, but the shape factor is set fixed across every level.

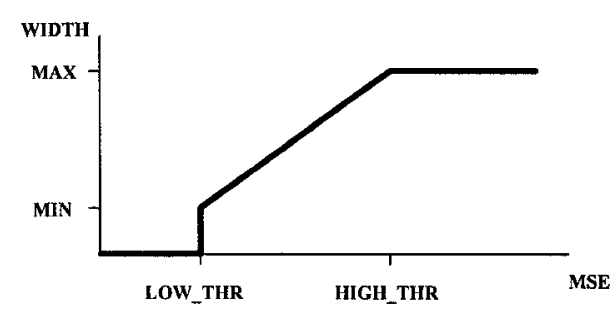

Fig.4. Normalized width function

\section{Experimental Results}

The results achieved by the proposed adaptive BBDE for stereoscopic video coding are satisfactory. Our approach can be used in a wide variety of application, from very low bitrates (where only the compensation scheme can be used, with a sparse field), to mid-range bitrates (in this case the field needs to be more accurate, and coding of the compensated frame difference (CFD) may be used). In any case, the adaptive BBDE performs better than the full search BBDE, in terms of computational load and better reconstruction quality, with the same number of disparity vectors. A comparison is given in Fig.5 for the stereo sequence "aqua" (256×256 pels).

\section{References}

1. D. H. Ballard and C. M. Brown, Computer Vision, Prentice-Hall, Englewood Cliffs, New Jersey 1982.

2. S. T. Barnard and M. A. Fischler, "Computational stereo", ACM Computing Surveys, Vol. 14, No. 4, pp. 553-572, December 1982.

3. F. Chassaing, B. Choquet and D. Pelé, "A stereoscopic television system (3D-TV) and compatible transmission on a MAC channel (3D-MAC)", Signal Processing: Image Communication, Vol. 4, No. 1, pp. 33-43, November 1991.

4. W. Li and E. Salari, "Efficient coding method for stereo image pairs", Visual Communications and Image Processing '93, Proc. SPIE 2094, pp. 1470-1476.

5. M. Bierling, "Displacement estimation by hierarchical block matching", Visual Communications and Image Processing '88, Proc. SPIE 1001, pp. 942-951.

6. F. Dufaux and M. Kunt, "Multigrid block matching motion estimation with an adaptive local mesh refinement", Visual Communications and Image Processing '92, Proc. SPIE 1818, pp. 97-109.

7. P.J. Burt and E. H. Adelson, "The Laplacian pyramid as a compact image code," IEEE Trans. Communications, Vol. 31, No. 4, pp. 532-540, April 1983.

8. P. Anandan, "A computational framework and an algorithm for the measurement of visual motion", International Journal of Computer Vision, Vol. 2, pp. 283-310, 1989. 


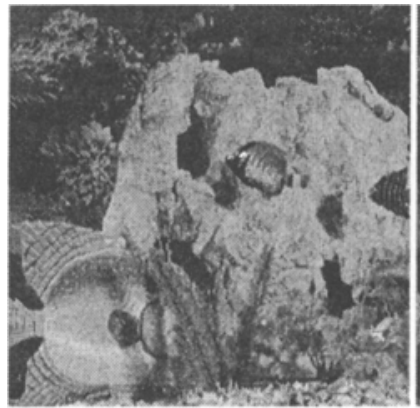

(a)

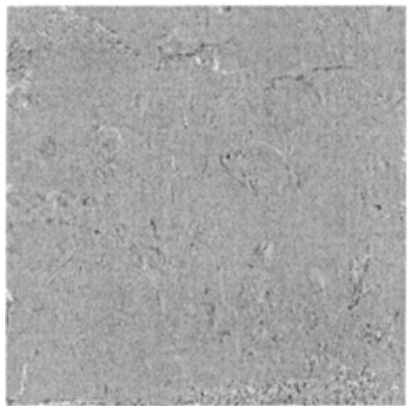

(b)

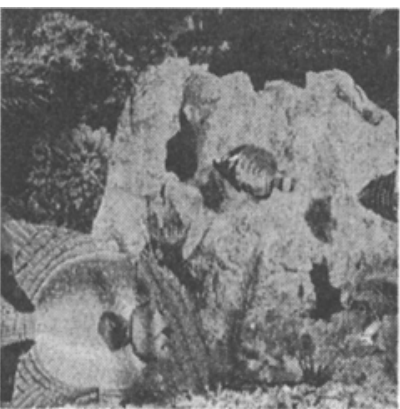

(d)

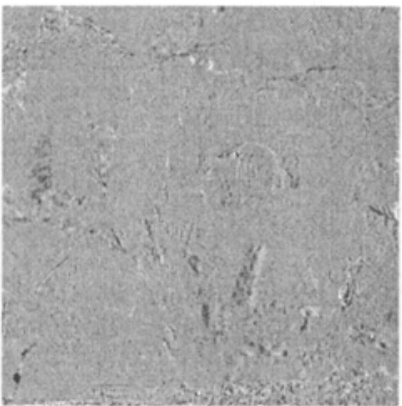

(e)

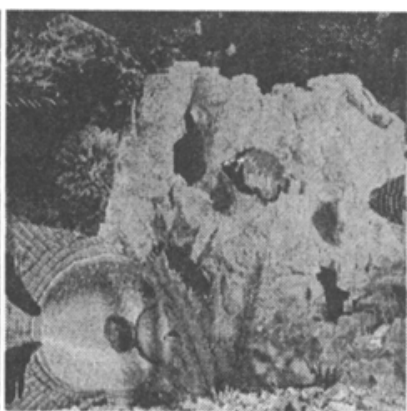

(g)

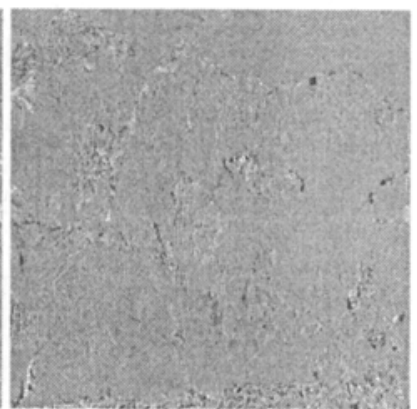

(h)

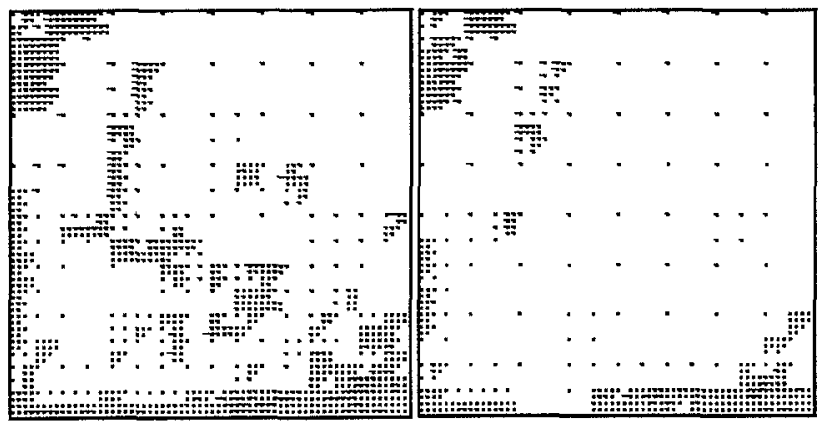

(c)

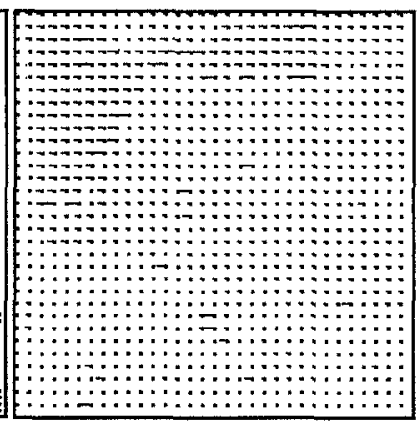

(i)

Fig.5. Experimental results:

$(\mathrm{a}, \mathrm{b}, \mathrm{c})$ : right frame reconstruction, CFD and needle diagram with adaptive $\mathrm{BBDE}(\mathrm{B}=4, \mathrm{MSE}=198,1024$ vectors$)$;

(d, e, f): right frame reconstruction, $C F D$ and needle diagram with adaptive $\mathrm{BBDE}(\mathrm{B}=4, \mathrm{MSE}=247,514$ vectors $)$;

$(\mathrm{g}, \mathrm{h}, \mathrm{i}): \quad$ right frame reconstruction, $\mathrm{CFD}$ and needle diagram with full-search fixed size $B B D E(B=8, M S E=248,1024$ vectors). 